\title{
Le neptunium : pourquoi de nouvelles études ?
}

\author{
F. PAQUET*, H. MÉTIVIER**, R. MASSE*
}

(Manuscrit reçu le 17 février 1994)

RÉSUMÉ Les activités développées au sein de l'industrie nucléaire vont engendrer de nouvelles manipulations d'actinides (programme SPIN). II importe, dans ce cadre, de définir pour chaque radionucléide utilisé un traitement en cas de contamination accidentelle. Le travail présenté ici fait le point des connaissances acquises dans le domaine des biocinétiques du neptunium, pour lequel aucun traitement efficace n'est aujourd'hui disponible. Des éléments de réflexion sont apportés, visant à développer les études réalisées jusqu'à présent, en leur imposant une approche expérimentale à un niveau subcellulaire et moléculaire.

ABSTRACT The development of new techniques in nuclear industry will involve increasing actinide manipulations. As a consequence, it is necessary to define a specific treatment in case of internal contamination. A review has been carried out of 50 references relating to the biological behaviour of neptunium and the decorporation efficiency of usual treatments. As a conclusion, there is no efficient therapeutics to remove neptunium after internal incorporation. New studies should now be developed, with particular attention focused on the behaviour of neptunium at the subcellular and molecular levels.

\section{Introduction}

Les activités développées au sein de l'industrie nucléaire peuvent conduire à une contamination accidentelle des individus par des éléments transuraniens. Dans un même temps, le développement du programme SPIN (séparation des actinides pour incinération) doit conduire à de nouvelles manipulations d'actinides et donc à un accroissement du risque d'incorporation. Parmi ceux ci, le neptunium est un élément important qui se rencontre principalement sous deux formes isotopiques, ${ }^{239} \mathrm{~Np}$ et ${ }^{237} \mathrm{~Np}$. Le premier $\left(T_{1 / 2}=2,36 \mathrm{j}\right)$ correspond à l'élément majoritaire mesuré dans l'environnement immédiatement après explosion nucléaire [33] alors que le second $T_{1 / 2}=2,2 \times 10^{6}$ ans) sera l'isotope prépondérant des déchets de haute activité dans quelques milliers d'années [5]. La biodisponibilité de cet élément, associée à sa manipulation dans de nombreuses industries nucléaires, en font un élément à risque en terme de radio-

* Commissariat à l'énergie atomique, Direction des sciences du vivant, Département de pathologie et de toxicologie expérimentale (CEA, DSV/DPTE), Laboratoire de Radiotoxicologie, BP 12, 91680 Bruyères le Châtel.

** Institut de protection et de sûreté nucléaire (IPSN), BP 6, F 92265 Fontenay-aux-Roses Cedex. 
protection. Les études de biocinétique de cet élément ont débuté en 1943 de façon très ponctuelle et ont eu un regain d'intérêt au début des années 1980 , avec la parution de nombreux travaux soviétiques résumés dans la revue de Thompson [47]. Ces études, associées à toutes celles qui ont suivi, ont largement décrit le métabolisme du radionucléide après administration et ont permis à la Commission internationale de protection radiologique (CIPR) de fixer des niveaux limites d'exposition [19]. Ces travaux ne sont malheureusement pas complets. A ce jour, les conclusions tirées se limitent à une description du cheminement du radionucléide à l'échelle de l'organe, et n'expliquent rien. Les données disponibles ne sont pas suffisantes pour comprendre les mécanismes de fixation du radionucléide au niveau moléculaire et la conséquence importante de cette lacune se traduit par une absence de traitement en cas de contamination accidentelle. Les études de décorporation expérimentale réalisées par Morin et al. [30] et Métivier (communication personnelle) ont montré l'inefficacité voire les méfaits d'une thérapeutique classique par administration de DTPA ou de diurétiques. Aujourd'hui, il est temps de lancer de nouvelles expérimentations afin de définir les ligands biologiques du neptunium et de tenter son élimination à l'aide de nouvelles molécules chélatrices. L'étude présentée ici fait le bilan des acquis dans ce domaine et propose de nouvelles voies d'investigation.

\section{Etat des travaux}

Les travaux effectués jusqu'à présent ont porté sur des études de biocinétique du radionucléide, de radiotoxicologie ou de décorporation $[19,47]$. Quelques études ont été réalisées chez l'homme [18, 35], mais l'essentiel des expériences a été réalisé chez le rat ou le singe.

\subsection{Biocinétique de l'élément neptunium}

\section{a) Absorption}

L'absorption gastro-intestinale de l'élément neptunium est comprise entre 0,1 et $1 \%$ de la masse ingérée. Ces valeurs sont sensiblement supérieures à celles des autres éléments transuraniens [1, 16, 31]. L'absorption dépend, toutefois, de nombreux paramètres physiologiques (espèce, âge de l'animal, état trophique) et physiques (forme physico-chimique du composé ingéré, masse et nature de l'alimentation,...) $[6,10,11,27,29,40-44,46,50]$. En particulier, il semblerait que l'absorption soit maximale chez les individus nouveau-nés (effet de l'âge), pour ${ }^{237} \mathrm{~Np}$ (effet de masse) et pour les formes oxydées $(\mathrm{Np}(\mathrm{VI})>\mathrm{Np}(\mathrm{V})>\mathrm{Np}(\mathrm{IV}))$. Des études physiologiques montrent qu'au niveau du tractus gastro-intestinal l'absorption se fait principalement au niveau de l'intestin grêle plutôt qu'au niveau de l'estomac [17].

Les études menées après contamination par inhalation montrent une très faible absorption pulmonaire, équivalente à environ $4 \%$ de la quantité dépo- 
sée dans le poumon $[1,45]$. Là encore, la forme physico-chimique du composé inhalé influe directement sur son transfert vers le sang. Malheureusement, lors des rares études réalisées, les auteurs ont souvent omis de préciser ces paramètres. Nombre de résultats sont ainsi difficilement exploitables.

\section{b) Organotropisme}

Les diverses contaminations expérimentales effectuées jusqu'alors ont montré, après transfert, une accumulation importante du radionucléide dans le squelette de l'animal. La charge squelettique est ainsi estimée à 50-70\% de la charge corporelle totale, en fonction du mode de contamination [19]. Cet ostéotropisme se traduit par une fixation du radionucléide sur les surfaces osseuses (principalement sur le périoste et sur les parties ostéogéniques) [37] ou par des agrégats sur la moelle osseuse [32].

Le foie concentre entre 5 et $10 \%$ de la radioactivité présente dans l'organisme. Quelques études effectuées au niveau subcellulaire semblent indiquer une fixation du radioélément dans les hépatocytes [47]. En fait, il semble que l'essentiel des échanges métaboliques ait lieu entre le foie et les os. Le rapport d'activité foie/os dépend du mode d'administration et de la forme initiale du composé. Après administration intrapéritonéale, intramusculaire ou après inhalation, ce rapport est faible. Inversement, après injection intraveineuse, ce rapport est beaucoup plus important [47]. De même, il semblerait que des administrations de neptunium sous forme d'oxalate ou de citrate entraînent une accumulation importante du radionucléide dans le foie, alors que des administrations de nitrate de neptunium conduisent à des charges osseuses plus importantes $[23,47]$.

Les autres organes de l'animal sont peu concernés par l'accumulation du neptunium. Les reins concentrent 1 à $4 \%$ du radionucléide et la rate présente toujours des taux inférieurs à $1 \%$.

\section{c) EIimination}

L'élimination du neptunium se fait principalement par voie urinaire. Cette forte composante s'expliquerait par le fait que Np reste en grande quantité sous forme libre dans le sang [30]. Thompson [47] rapporte que le tiers de la concentration plasmatique de ${ }^{237} \mathrm{~Np}$ ou de ${ }^{239} \mathrm{~Np}$ est ainsi éliminé en $24 \mathrm{~h}$. Cette forme d'élimination est très différente de ce qui est observé avec les autres éléments transuraniens. En règle générale, ces derniers sont éliminés principalement sous forme fécale.

\section{d) Cinétiques d'échanges}

La décorporation naturelle du neptunium est faible. Ballou et al. [1] montrent que, 3 semaines après administration intraveineuse de citrate de ${ }^{237} \mathrm{~Np}$, $43 \%$ de la charge initiale restent dans l'organisme. Les cinétiques d'élimination dépendent, toutefois, de l'organe considéré et du mode d'administration. Thompson [47] distingue des organes comme les reins où l'élimination se fait 
avec des périodes biologiques égales à $80 \mathrm{j}$ et d'autres comme les glandes surrénales où l'élimination est nulle. De façon générale, après administration intraveineuse de nitrate de neptunium, il semblerait que l'élimination du composé se fasse selon 3 composantes, correspondant à autant de compartiments biologiques : $40 \%$ du radionucléide seraient éliminés avec une période biologique égale à $5 \mathrm{j}, 15 \%$ avec une période de 5 mois et $45 \%$ avec une période de 3,5 ans. Ce dernier compartiment correspondrait à l'élimination osseuse [50].

\subsection{Toxicologie du neptunium}

La toxicité du neptunium peut être d'origine chimique ou radiologique. En fait, la nature des effets nocifs est surtout liée à la nature de l'isotope incriminé. De par ses caractéristiques (activité massique $=2,55 \times 10^{7} \mathrm{~Bq} \mathrm{~g}^{-1} ; \mathrm{T}_{1 / 2}=2,2 \mathrm{x}$ $\left.10^{6} \mathrm{ans}\right),{ }^{237} \mathrm{~Np}$ est le dernier actinide à présenter une toxicité chimique.

\section{a) Toxicité chimique}

Pour ${ }^{237} \mathrm{~Np}$, des effets létaux sont observés après administration de doses, variables suivant les organismes, comprises entre 6 et $24 \mathrm{mg} \mathrm{kg}^{-1}$ selon l'espèce et le sexe considéré $[3,25]$. Après administration de doses létales, les autopsies pratiquées sur les animaux ont permis de montrer des lésions au niveau du foie, des reins, de la rate ou du cerveau [2,24]. Des hémorragies cérébrales ont également été rapportées dans quelques cas [31]. Quelques effets sublétaux ont été observés à partir de concentrations égales à $1 \mathrm{mg} \mathrm{kg}^{-1}$ et se sont traduits par des baisses d'appétit, des asthénies ou des changements de composition plasmatique [21]. Cette toxicité chimique observée expérimentalement est liée, toutefois, aux masses importantes de neptunium administrées; elle est donc peu probable en cas d'exposition accidentelle.

\section{b) Toxicité radiologique}

Les effets radiologiques s'observent, en général, après contamination par ${ }^{239} \mathrm{~Np}$ ou après de très longues expositions à ${ }^{237} \mathrm{~Np}$. Les principales lésions observées sont des ostéosarcomes, en raison du tropisme osseux de l'élément, ou des tumeurs pulmonaires lorsque l'administration est intratrachéale [23].

\subsection{Principaux acquis dans le domaine du traitement des contaminations au neptunium}

Le traitement applicable pour la décorporation des éléments transuraniens s'articule sur deux principes complémentaires :

- La première action doit aboutir à une réduction du transfert du radionucléide au niveau du point d'entrée. Ce principe s'applique surtout dans le cas d'éléments transférables, où l'absorption et le transfert du radionucléide sont, en général, très rapides. Dans ce cas, le traitement doit intervenir le plus tôt possible et peut consister, en cas de contamination par ingestion, en l'adjonction d'absorbants et d'antiacides, bien qu'ils ne présentent qu'une faible effica- 
cité. Dans le cas de formes difficilement transférables, les éléments ne participent pas aux mécanismes biologiques et franchissent faiblement les barrières biologiques. En cas d'inhalation accidentelle de formes non solubles (cas des oxydes), le traitement peut être le lavage pulmonaire. Celui-ci est envisagé dans les cas de forte contamination $(>100 \mathrm{LAI})$ et lorsque les bénéfices à retirer pour le patient sont supérieurs aux risques engendrés par l'opération (en fonction de paramètres médicaux et psychosociologiques) [4].

- Le deuxième principe consiste à augmenter l'excrétion du radionucléide. La technique la plus efficace est d'administrer un agent chélatant, capable de se substituer au ligand biologique et de permettre l'élimination du radionucléide par les voies naturelles.

La première approche dans le traitement des contaminations au neptunium a été de tester l'efficacité des procédés traditionnellement mis en œuvre dans le cas d'administration d'autres éléments transuraniens. Diverses molécules ont été testées dans le cadre de protocoles déjà établis pour la décorporation du plutonium notamment. Une des molécules les plus utilisées a été le DTPA. Cette molécule forme des complexes stables avec bon nombre d'éléments transuraniens, et devait permettre une décorporation efficace. Les essais de décontamination du neptunium après exposition expérimentale et administration de DTPA ont montré la limitation de la méthode. En fait, il semblerait, selon Morin et al. [30], que le complexe Np-DTPA soit instable dans le plasma et conduise plutôt à une augmentation de la charge osseuse. Les diurétiques employés par Métivier (communication personnelle) conduisent aux mêmes résultats. A notre connaissance, peu d'études ont été effectuées, mais toutes montrent que les agents complexants habituellement utilisés à des fins de décontamination se révèlent inefficaces dans le cas du neptunium [30, 47-48]. Les études les plus poussées ont tenté de réduire la charge corporelle de neptunium, chez un rat contaminé, par administration de plusieurs agents complexants, dont le DTPA, le LICAM et le DFOA. L'idée de base était que le neptunium plasmatique, se fixant en partie sur la transférine [49], devait être déplacé par des agents complexants spécifiques du fer. Les conclusions essentielles à tirer de ces travaux sont au nombre de trois :

- la chélation du neptunium in vivo ne peut avoir lieu que sous sa forme tétravalente ; dans l'organisme, il semble qu'une partie du neptunium soit chélatable et l'autre non;

- le DTPA n'agit sur la décorporation du neptunium que lorsqu'il est administré à forte concentration (le rapport massique DTPA/Np doit être supérieur à $10^{9}$ ) ; ceci implique l'utilisation de doses toxiques pour l'organisme ; l'utilisation de DTPA aux doses usuelles de $30 \mu \mathrm{g} / \mathrm{kg}$ (soit $1 \mathrm{~g}$ pour un homme de $70 \mathrm{~kg}$ ) ne réduit la charge corporelle que de quelques pour cent;

- l'action d'un agent utilisé seul peut entraîner une concentration tissulaire locale du radionucléide ; c'est le cas du LICAM pour les reins et du DTPA pour les os $[15,48]$; en fait, l'efficacité des drogues utilisées est plus importante lorsque celles-ci sont utilisées en synergie $[12,13,28]$. 
En d'autres termes; et en conclusion, il n'existe, au stade actuel, aucun traitement applicable pour la décorporation du neptunium.

\section{Etudes à réaliser}

La rapide énumération des résultats de travaux portant sur les biocinétiques et la toxicologie du neptunium soulève un certain nombre d'inconnues. Les études effectuées jusqu'à ce jour sont restées très éparses et peu approfondies. En particulier, de nombreux travaux restent à effectuer avant d'élucider les mécanismes de transfert du radioélément. De façon générale, deux domaines restent à explorer :

- l'étude des ligands systémiques et intracellulaires du neptunium;

- la mise au point d'une thérapeutique à appliquer après contamination.

\subsection{Détermination des ligands biologiques du neptunium}

Les quelques études décrites ci-dessus relatent l'importance de l'état de valence de la forme administrée. Il est indispensable de compléter les études réalisées par des travaux faisant intervenir de façon spécifique l'état d'oxydation du radionucléide. De plus, de nouvelles méthodes analytiques, imposant une approche à un niveau moléculaire, doivent permettre, à terme, de donner des indications sur la nature des mécanismes de fixation du radionucléide sur les ligands biologiques. Cette approche du problème n'a été abordée jusqu'à présent que dans très peu d'études $[14,22,26,36,49]$ et devrait être largement complétée. Il s'agirait alors de déterminer les sites accepteurs des ligands biologiques, ainsi que les cinétiques d'échanges entre ces sites. Ces études impliqueraient des phases de travail in vivo (localisation et identification des sites accepteurs du radionucléide) et in vitro (cinétiques de complexation et de décomplexation, compétition avec divers agents,...) par des techniques empruntées à la biochimie (chromatographie hydrophobe et en gel filtration, ultrafiltration, ultracentrifugation, précipitations sélectives,...). Cette approche plus fondamentale du problème, axée principalement sur la détermination des processus chimiques régissant les phénomènes de fixation du radioélément, nous donnera de précieuses indications sur les mécanismes mis en ouvre et donc sur les traitements à effectuer.

\subsection{Traitements}

Les traitements associés à la décorporation du neptunium peuvent être de deux ordres. La première approche consiste évidemment à rechercher un agent capable de former avec le neptunium un complexe éliminé par l'organisme par voie urinaire. Le complexe doit être suffisamment stable et doit être capable d'agir au niveau intra-cellulaire [20]. Les études effectuées avec le DTPA montrent que le neptunium réagit différemment des autres éléments transuraniens. 
Cela montre la nécessité de définir l'état d'oxydation du radionucléide in vivo et, le cas échéant, de le réduire à l'état $\mathrm{Np}(\mathrm{IV})$. De plus, il serait intéressant de définir d'autres molécules chélatrices ayant une plus grande affinité pour le radionucléide. La panoplie des chélateurs existants est large et peut être utilisée à souhait. En dehors des molécules dérivées ou analogues (DTPA-DX, EDTA, HEDTA), des études ont été réalisées avec des molécules analogues des sidérophores, capables de complexer spécifiquement le fer. Des essais ont ainsi été réalisés avec le LICAM et le DFOA [48]. Ces composés ne donnent pas de résultats excellents mais l'action doit être poursuivie. La fixation, même partielle, du neptunium sur la transférine laisse penser que le radionucléide suit le métabolisme du fer et peut donc être complexé par ces molécules. Des essais de décorporation ont été tentés pour le plutonium et l'américium avec le 3,4,3-LIHOPO. Ces deux radionucléides se fixent également sur la transférine et les résultats de décorporation sont très satisfaisants [8, 34, 38-39]. Les capacités de complexation de cette molécule semblent être largement supérieures à celles des autres molécules testées (3,4,3-LICAM, DFO-HOPO, DTPA-DX) mais aucune étude avec le neptunium n'a encore été menée. Pourtant, son action sur le radionucléide semble être certaine [9]. Cet agent devra donc être testé à la fois in vivo et in vitro sur des rongeurs et des primates non humains.

L'autre voie d'action qui a été envisagée pour la décorporation des éléments transuraniens est l'utilisation de diurétiques, chargés d'augmenter l'excrétion urinaire. Des essais ont été effectués par Dilley [7] qui montraient que l'acétazolamide (DIAMOX) ou le chlorothiazide (DIURILIX) rendaient le plutonium inhalé soluble et chélatable par le DTPA. D'autres expérimentations réalisées par Métivier (communication personnelle) montrent, au contraire, que le DIAMOX ou le LASILIX ont pour effet d'augmenter la charge osseuse en neptunium chez des singes contaminés. D'autres études sont donc à effectuer avant de conclure au rôle de ces médicaments.

\section{Conclusion}

Le bilan des études réalisées avec le neptunium montre la nécessité de développer certains aspects complètement négligés jusqu'alors. L'intérêt de relancer de telles études ne tient pas tant dans un besoin de recherche de base que par la nécessité qu'il y a de pouvoir répondre aux éventuelles contaminations dues à cet élément, dont la manipulation ne cesse de croître. La voie à privilégier consiste à définir, en premier lieu, la forme physico-chimique du rạdionucléide in vivo, puis à tester de nouvelles molécules chélatrices. Les progrès réalisés en biochimie et en biologie moléculaire doivent apporter les outils nécessaires à de telles études.

\section{Remerciements}

Cette étude s'inscrit dans le cadre d'un projet financé par l'Institut de protection et de sûreté nucléaire (IPSN). 


\section{RÉFÉRENCES}

[1] BAlloU J.E., BAIR W.J., CASE A.C., THOMPSON R.C. - Studies with neptunium in the rat. Health Phys., 1962, 8, 685-688.

[2] BERLIN J.D., MAHLUM D.D. - Comparative morphologic and functional studies of neptunium induced fatty livers in rats. Lab. Invest., 1970, 22, 252-259.

[3] CASEY H.W., McClellaN R.O., CLARKE W.J., BUSTAD L.K. - Acute toxicity of neptunium-237 and its relationship to liver function in sheep. Health Phys., $1963,9,827-83$.

[4] COMMISSION OF THE EUROPEAN COMMUNITIE (CEC), US DEPARTMENT OF ENERGY. - Guidebook for the treatment of accidental radionuclide contamination of workers. Radiat. Prot. Dosim, 1992, 41, 1-50.

[5] COHEN B.L. - Effects of ICRP publication 30 and the 1980 BEIR report on hazard assessments of high-level waste. Health Phys., 1982, 42,133-143.

[6] DAVID A.J., HARRISON J.D. - The absorption of ingested neptunium, plutonium and americium in newborn hamsters. Int. J. Radiat. Biol., 1984, 46, 279-286.

[7] DILLEY J.V. - Therapeutic removal of inhaled plutonium. Rapport BNWL-1306, Part 1. Pacific Northwest Laboratory Annual, Report for 1969 to USAEC, 1970.

[8] DURBIN P.W. - The efficacies of 3,4,3-LIHOPO and DTPA for enhancing the excretion of plutonium and americium from the rat : comparison with other siderophore analogues. Int. J. Radiat. Biol., 1992, 62, 487-497.

[9] DURBIN P.W., KULLGREN B., XU J., RAYMOND K.N. - Efficient in vivo chelation of $\mathrm{Pu}(\mathrm{IV}), \mathrm{Am}(\mathrm{III})$ and $\mathrm{Np}(\mathrm{V})$ in mice by TREN-(3,2-HOPO). Radiat. Prot. Dosim. (à paraître).

[10] FRITSCH P., BEAUVAlLET M., JOUNIAUX B., METIVIER H., MASSE R. Transfer of $\mathrm{Np}(\mathrm{V})$ nitrate from gastrointestinal segments of the adult rat. Int. J. Radiat. Biol., 1986, 50, 609-620.

[11] FRITSCH P., BEAUVALleT M., JOUNIAUX B., MOUTAIROUS K., METIVIER H., MASSE R. - Effects of the chemical forms and valency states of neptu- nium on its jejunal transfer in the rat. Int. J. Radiat. Biol., 1987, 52, 505-515.

[12] GACHALYI A., NAMENYI J., SZEGEDI I., VARGA L.P. - Influence of mixed ligand complexes on retention and distribution of radioniobium in mice. Radiat. Res., 1987, 112, 312-317.

[13] GACHALYI A., NAMENYI J., SZEGEDI I., VARGA L.P. - Effect of mixed therapy on the retention of ${ }^{95} \mathrm{Nb}$ and ${ }^{144} \mathrm{Ce}$ in mice. Radiat. Res., 1989, 120, 177-181.

[14] GALLE P., BOULHADOUR H., METIVIER H. - Selective localization of neptunium-237 in nuclei of mammalian cells. C.R. Acad. Sci. III, 1992, 314,1-5.

[15] GERASIMO P., DUSERRE C., METIVIER H. - Biological behaviour of Pu administered to animals as Pu-standard LICAM(C) complex : therapeutical attempts to decrease Pu kidney burden. Human Toxicol., 1986, 5, 309-318.

[16] HARRISON J.D. - The gastrointestinal absorption of the actinide elements. Sci. Total Environ., 1991, 100, 43-60.

[17] HARRISON J.D., POPPLEWELL D.S., DAVID A.J. - The effect of concentration and chemical form on the gastrointestinal absorption of neptunium. Int. J. Radiat. Biol., 1984, 46, 269-277.

[18] HARRISON J.D., POPPlEWEll D.S., ETHERINGTON G., HAM G.J. Gastrointestinal absorption of neptunium and curium in volunteers. Radiol. Prot. Bull., 1988, 93, 11-14.

[19] INTERNATIONAL COMMISSION ON RADIOLOGICAL PROTECTION (ICRP). - Age-dependent doses to members of the public from intake of radionuclides, Part 1 (ICRP Publication 56). Oxford : Pergamon press, 1989, Ann. ICRP, 20, 69-73. 
[20] JONES M.M. - The design of therapeutic chelating agents. J. Coord. Chem., 1991, $23,187-200$.

[21] KRESLOV V.V., MAKSUTOVA A.Y., MUSHKACHEVA G.S. - Behaviour of neptunium-237 in subcellular fractions of rat kidney. Radiobiologiya, 1978, 18, 84-88.

[22] KRESLOV V.V., MUSHKACHEVA G.S. - Change in composition of rat blood as affected by ${ }^{237} \mathrm{~Np}$. Ukr. Biokhim. Zh., 1970, 42, 488-493.

[23] LEVDIK T.I., LEMBERG V.K., BULDAKOV L.A., LYUBCHANSKII E.R., PESTERNIKOV V.M.- Biological effectiveness of ${ }^{237}$ Np. Health Phys., 1972, 22, 643645.

[24] MAHLUM D.D. - Modification of the hepatotoxic action of neptunium-237 in the rat. Toxicol. Appl. Pharmacol., 1971, 18, 696-699.

[25] MAHLUM D.D., CLARKE W.J. - Neptunium-237 toxicity in the rat. I. Histopathologic and chemical observations in liver and kidney. Health Phys., 1966, 12, 713.

[26] MASSE R., JOVANOVIC V., FRAGU P., L'HULliER I., LAFUMA J. Utilisation de l'electrophorèse en rideau pour la détermination de l'état physicochimique des radioéléments et des composés radioactifs en solution dans les liquides biologiques : fer, cérium, neptunium, calcitonine en solution. Rapport CEA-R-4417, 1973.

[27] METIVIER H., BOURGES J., FRITSCH P., NOLIBE D., MASSE R. - Gastrointestinal absorption of neptunium in primates : effects of ingested mass, diet, and fasting. Radiat. Res., 1986, 106, 190-200.

[28] METIVIER H., DUSERRE C., GERASIMO P., FRITSCH P., MASSE R. - Essais de décontamination après inhalation par des babouins du complexe plutonium-tributylphosphate. Comparaison DTPA-LICAM (C). In : Radiation protection practice, $7^{\text {th }}$ international congress of the International radiation protection association, Sidney, April 10-17, 1988. Oxford : Pergamon Press, 1988, 3, 1196-1199.

[29] METIVIER H., MASSE R., LAFUMA. - Effet de la masse sur l'absorption gastrointestinale du neptunium $\mathrm{V}$ chez le singe et le rat. Radioprotection, 1983, 18, 13-17.

[30] MORIN M., NENOT J.C., LAFUMA J. - The behaviour of ${ }^{237} \mathrm{~Np}$ in the rat. Health Phys., 1973, 24, 311-315.

[31] NENOT J.C. - The metabolism and toxicity of neptunium. In : Radionuclides, metabolism and toxicity (GALLE P., MASSE R., Eds.), Paris : Masson, 1982, 225-231.

[32] NENOT J.C., MASSE R., MORIN M., LAFUMA J. - An experimental comparative study of the behaviour of ${ }^{237} \mathrm{~Np},{ }^{238} \mathrm{Pu},{ }^{239} \mathrm{Pu},{ }^{241} \mathrm{Am}$, and ${ }^{242} \mathrm{Cm}$ in bone. Health Phys., 1972, 22, 657-665.

[33] PERKINS R.W., THOMAS C.W. - Worldwide fallout. In : Transuranic elements in the environment (W.C. HANSON, Ed.), Oak Ridge, Tenn. : USDOE Technical information center, 1980, 53-82 (DOE-TC-22800).

[34] PONCY J.L., RATEAU G., BURGADA R., BAILlY T., LEROUX Y., RAYMOND K.N., DURBIN P.W., MASSE R. - Efficacy of 3,4,3-LIHOPO for reducing the retention of ${ }^{238} \mathrm{Pu}$ in rat after inhalation of the tributylphosphate complex. Int. J. Radiat. Biol., 1993, 64, 431-436.

[35] POPPLEWELL D.S., HARRISON, J.D., HAM G.J. - Gastrointestinal absorption of neptunium and curium in humans. Health Phys., 1991, 60, 797-805.

[36] SEIDEL D., WIENER M., KRUEGER E., WIRTH R., HAFFNER H. - Studies on the lysosomal binding of ${ }^{141} \mathrm{Ce},{ }^{239} \mathrm{~Np},{ }^{239} \mathrm{Pu}$ and ${ }^{241} \mathrm{Am}$ in rat and syrian hamster liver using carrier-free electrophoresis. Nucl. Med. Biol., 1986, 13, 515-518.

[37] SONTAG W. - Microdistribution of ${ }^{237} \mathrm{~Np}$ in the skeleton of female rats. Int. J. Radiat. Biol., 1993, 63, 383-393. 
[38] STRADLING G.N., GRAY S.A., MOODY J.C., HODGSON A., RAYMOND K.N., DURBIN P.W., RODGERS S.J., WHITE D.L., TUROWSKY P.N. - The efficacy of DFO-HOPO, DTPA-DX and DTPA for enhancing the excretion of plutonium and americium from the rat. Int. J. Radiat. Biol., 1991, 59, 1269-1277.

[39] STRADLING G.N., GRAY S.A., ELLENDER M., MOODY J.C., HODGSON A., PEARCE M., WILSON I., BURGADA R., BAILLY T., LEROUX Y., EL MANOUNI D., RAYMOND K.N. - The efficacies of 3,4,3-LIHOPO and DTPA for enhancing the excretion of plutonium and americium from the rat : comparison with other siderophore analogues. Int. J. Radiat. Biol., 1992, 62, 487-497.

[40] SULLIVAN M.F. - Absorption of actinides elements from the gastrointestinal tract of neonatal animals. Health Phys., 1980, 38,173-185.

[41] SULLIVAN M.F., GORHAM L.S. - Further studies on the absorption of actinides elements from the gastrointestinal tract of neonatal animals. Health Phys., 1982, 43, 509-519.

[42] SULLIVAN M.F., MILLER B.M., RYAN J.L. - The effect of mass on gastrointestinal absorption of plutonium and neptunium. Radiat. Res., 1983, 94,199-209.

[43] SULLIVAN M.F., MILLER B.M., RUEMMLER P.S. - Further studies on the influence of chemical form and dose on absorptions of $\mathrm{Np}, \mathrm{Pu}, \mathrm{Am}$ and $\mathrm{Cm}$ from the gastrointestinal tracts of adult and neonatal rodents. Health Phys., 1985, 48, 61-73.

[44] SULLIVAN M.F., RUEMMLER P.S. - Absorption of uranium 233, neptunium 237, plutonium 238, americium 241 and curium 244 from the gastrointestinal tracts of the rats fed an iron-deficient diet. Health Phys., 1988, 54, 311-316.

[45] SULLIVAN M.F., RUEMMLER P.S., BUSCHBOM R.L. - Neptunium 237 inhalation in rats. Health Phys., 1986, 51, 745-753.

[46] SULLIVAN M.F., RUEMMLER P.S., RYAN J.L. - Effects of fasting and/or oxidizing and reducing agents on absorption of neptunium from the gastrointestinal tract of mice and adult or neonatal rats. Radiat. Res., 1984, 100, 519-526.

[47] THOMPSON R.C. - Neptunium - the neglected actinide : a review of the biological and environmental literature. Radiat. Res., 1982, 90, 1-32.

[48] VOLF V., WIRTH R. - Effective chelation therapy after incorporation of neptunium-239 in rats. Int. J. Radiat. Biol., 1986, 50, 955-959.

[49] WIRTH R., TAYLOR D.M., DUFFIELD J. - Identification of transferrin as the principal neptunium binding protein in the blood serum of rats. Int. J. Nucl. Med. Biol., 1985, 12, 327-330.

[50] WIRTH R., VOLF V. - Long term retention of ${ }^{237} \mathrm{~Np}$ in rats. Int. J. Radiat. Biol., $1984,46,787-792$. 